\title{
Septic Arthritis of the Pediatric Shoulder: From Infancy to Adolescence
}

\author{
Justin W. Walker ${ }^{1}$ and William L. Hennrikus ${ }^{1,2}$ \\ ${ }^{1}$ College of Medicine, The Pennsylvania State University, Hershey, PA 17033, USA \\ ${ }^{2}$ Penn State Hershey Medical Center, Bone and Joint Institution, Hershey, PA 17033, USA
}

Correspondence should be addressed to Justin W. Walker; jwalker7@hmc.psu.edu

Received 19 May 2016; Accepted 25 July 2016

Academic Editor: Steven E. Lipshultz

Copyright (c) 2016 J. W. Walker and W. L. Hennrikus. This is an open access article distributed under the Creative Commons Attribution License, which permits unrestricted use, distribution, and reproduction in any medium, provided the original work is properly cited.

\begin{abstract}
Background. Septic arthritis of the glenohumeral joint in the pediatric population $(<18$ yo $)$ is not commonly described in the literature. There is a corresponding paucity of information regarding its presentation and treatment. Methods. An IRB approved review of patients treated with irrigation and debridement by the Orthopaedic Department was completed. This retrospective study includes four patients, presenting from 2005 to 2015, with septic arthritis of the shoulder. Results. The mean age $\left(M_{\text {age }}\right)$ at presentation was 5 years, with a range of 1 month to 15 years. Patients presented on average after 7 days with pain and a mean temperature of $39^{\circ} \mathrm{C}$, erythrocyte sedimentation rate of $66 \mathrm{~mm} / \mathrm{hr}$, a C-reactive protein level of $11.17 \mathrm{~g} / \mathrm{dL}$, and a white blood cell count of $20.2 \times 10^{3} / \mathrm{mcL}$. Staphylococcus aureus, Candida albicans, and Pseudomonas aeruginosa were cultured from the wounds. All cases were treated operatively with irrigation and debridement and with antimicrobial therapy. Patients received antibiotics for an average of 6 weeks. Conclusion. Septic arthritis of the shoulder occurs in all pediatric ages. Successful treatment of septic arthritis of the shoulder was accomplished in four cases without division of the biceps sheath, with an average follow-up of 8 months.
\end{abstract}

\section{Introduction}

Septic arthritis of the pediatric shoulder is a rare condition, with most reports citing a prevalence of $3-5 \%$ of all septic joints [1]. As such, there is a lack of data available regarding its clinical presentation, clinical course, and treatment. Additionally, septic arthritis of the shoulder is often stated to be a disease of infancy and is commonly associated with osteomyelitis of the adjacent bone [1-3]. The anatomy of the shoulder joint likely contributes to the association with osteomyelitis, as the capsule of the shoulder envelopes the metaphysis, facilitating hematogenous spread between the bone and joint space [2]. Treatment recommendations vary; however, surgical opening and exploration of the glenohumeral capsule and biceps tendon sheath are recommended for thorough cleansing of the joint [4]. In this review, 42 children with septic joints were identified, of whom 4 , or $9.5 \%$, were found to have septic arthritis of the shoulder.

\section{Materials and Methods}

Following medical school Institutional Review Board (IRB) approval, review of all records, from 2008 to 2015, for pediatric patients $(<18$ yo $)$ with septic shoulders treated with irrigation and debridement was completed. Forty-two patients with septic arthritis were identified, of whom 5 had an infected shoulder. One of the five was a 17-year-old female who acquired a shoulder infection as a postoperative complication and was excluded from the current study $(N=4)$. Special attention was paid to the time required for diagnosis, laboratory data, treatment strategies, and long-term complications.

\section{Results}

Seventy-five percent of patients in this study were male. The average age was 5 years with a range of 1 month to 15 years. 
The average time until diagnosis was 7 days, with a range of 4 to 10 days. All four patients presented with decreased use of the affected shoulder, and 1 of the 4 had an antecedent trauma (case 2). This patient fell onto the affected shoulder during a basketball game "a few weeks" prior to presentation. Two patients were born prematurely at 27 weeks (case 3 ) and 32 weeks (case 4 ). See Table 1 for a synopsis of cases.

Three of 4 patients had elevated C-reactive protein (CRP) levels on laboratory data with an average of $11.17 \mathrm{~g} / \mathrm{dL}(0.8-$ $14.3 \mathrm{~g} / \mathrm{dL})$. The erythrocyte sedimentation rate (ESR) was reported in 3 of the 4 patients, with an average of $66 \mathrm{~mm} / \mathrm{hr}$ $(20-125 \mathrm{~mm} / \mathrm{hr})$. The average temperature was $39.0^{\circ} \mathrm{C}$. The white blood cell count (WBC) had an average of $20.19 \times$ $10^{3} / \mathrm{mcL}(17,000-23,800)$.

A positive culture from synovial fluid was obtained in $100 \%$ of patients. These consisted of methicillin-resistant Staphylococcus aureus (two), methicillin-sensitive Staphylococcus aureus, Candida albicans, and Pseudomonas aeruginosa. One patient's culture grew both Candida albicans and methicillin-sensitive Staphylococcus aureus.

One patient was treated with an aspiration followed by arthrotomy and antibiotics, while the other three were treated with arthrotomy and antibiotics. In all surgeries, the biceps tendon sheath was left intact. Patients were treated with antibiotics for an average of 43 days, with a range of 32 to 48 days. In 3 of 4 cases, both parenteral and oral antibiotics were used. Empirical treatment with vancomycin was initiated in all four cases until culture returned and a more narrowspectrum antibiotic could be chosen.

The average follow-up of these patients was 8 months, with a range of 2 months to 12 months. Of these patients, one reported decreased range of motion at 2 months of follow-up (case 1).

\section{Discussion}

Septic arthritis of the glenohumeral joint is considered by many to be a disease of infancy $[1,2,5,6]$. However, in this study, only 2 patients were infants $\left(M_{\text {age }}=5\right.$ years $)$. Each patient presented to the emergency department, three as transfers from an outside institution and one from home. In each case, the patient's chief complaint included pain with motion in the glenohumeral joint. In case 3, the patient also presented with decreased movement of the right lower extremity secondary to a concomitant knee infection.

The initial diagnosis of septic arthritis requires astute clinical judgment. Literature regarding the initial evaluation of children with joint pain or concern for septic arthritis recommends obtaining a full blood count with differential, ESR, CRP, synovial fluid analysis, and synovial fluid culture [6-9]. Of the laboratory studies done in this study, the most consistently elevated marker was total white blood cell count $(>12,000)$, which was present in $100 \%$ of the cases. The ESR and CRP were elevated ( $>2 \mathrm{~g} / \mathrm{dL}$ and $>40 \mathrm{~mm} / \mathrm{hr}$, resp.) in $75 \%$ of the cases. However, the ESR was not requested in one of the cases, likely due to the patient's age ( 3 months). The elevated CRP in $75 \%$ of the cases supports recent literature recommending the inclusion of CRP in the diagnostic criteria for septic arthritis [8].

Cultures obtained from these cases were positive for MRSA (2), MSSA (1), Pseudomonas aeruginosa (1), and Candida albicans (1). One of the cases had coinfection with both MSSA and C. albicans. One of 4 patients was bacteremic, and another was both bacteremic and fungemia.

The fungemia patient, 4-month-old born at 27-week gestation, had multifocal septic arthritis, with positive cultures for Candida albicans and MSSA found in both the glenohumeral and acetabulofemoral joints. The severity of this presentation and pathogen involved can be explained partly by the increased susceptibility of infant's joints to hematogenous spread of infection, as well as the immature immune system associated with infants of preterm birth $[5,10,11]$.

Fifty percent of cases in this review presented with concomitant osteomyelitis and septic arthritis of the shoulder. These data are similar to the reported rate of $75 \%$ by Montgomery et al., $67 \%$ by Belthur et al., and $56 \%$ by Schmidt et al. $[3,9,12]$. In a separate study, it was noted that $100 \%$ of patients $(N=6)$ with osteomyelitis of the proximal humerus had septic arthritis of the shoulder [4]. In all cases of osteomyelitis in the current study, the metaphysis was drilled in order to decompress the bone, in keeping with previous recommendations [2].

In addition to laboratory evaluation, imaging modalities are recommended in the evaluation of septic arthritis $[1,2,4]$. In all 4 cases, imaging modalities were used to assist in the diagnosis of septic arthritis, with MRI being the modality of choice. Initial plain radiographs were obtained in all four cases and failed to note abnormalities in $75 \%$ of the patients. In addition, plain radiograph had a false-negative rate of $50 \%$ for changes consistent with osteomyelitis. Technetiumpyrophosphate bone scans have been reported as useful in the literature, as they assist in the diagnosis of skip lesions in osteomyelitis, which are often missed when localized MRIs are used [13]. Bone scans were not used in the diagnosis of the patients in this series.

Treatment of septic arthritis of the shoulder includes appropriate antibiotic therapy and drainage. Infectious disease consult is prudent and was done in all 4 cases. Repeat aspiration and irrigation and debridement are reported modalities of drainage for septic arthritis of the shoulder [14]. However, one author recommends dividing and exploring the biceps tendon sheath to ensure full debridement of infected tissue [3]. In the current study, the surgical exploration and treatment left the biceps tendon intact, with a thorough irrigation of the joint noted in each procedure.

On follow-up, the patients' outcomes were generally good. None of the patients had long-term clinical deficits. The patient in case 2 was noted to have sclerotic changes to the proximal humerus on radiograph, without clinical manifestations of these changes. This patient was able to return to basketball at a collegiate level.

Previous studies demonstrated a delay in treatment for shoulder infection as compared to hip infections and postulated that the lack of an evidence-based clinical algorithm may contribute [9]. The Kocher criteria were developed as a joint-specific algorithm for the diagnosis of septic arthritis 


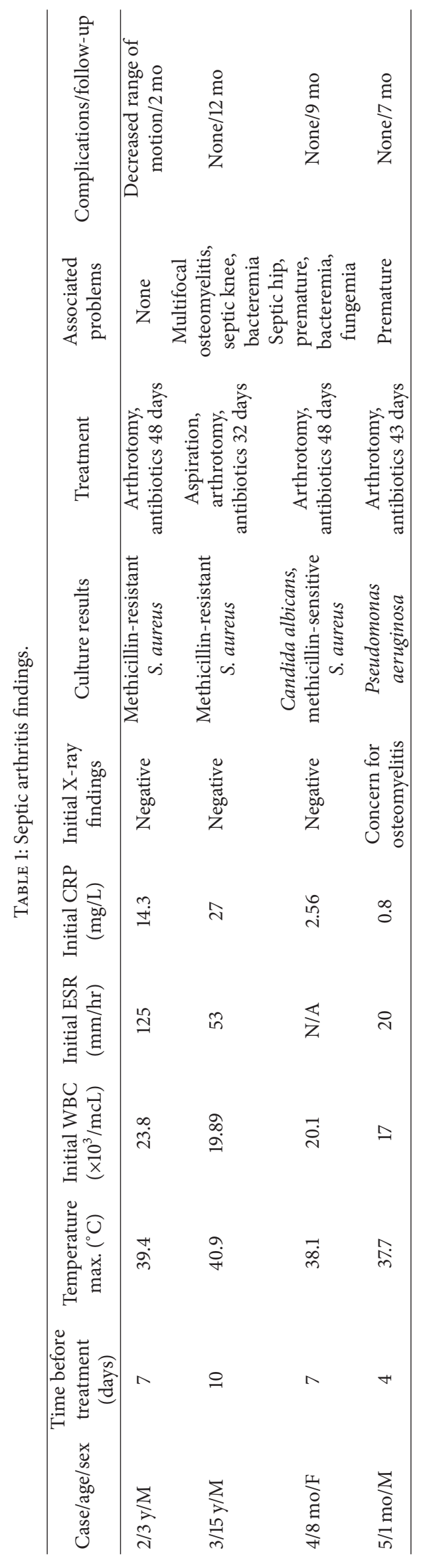


of the pediatric hip and have facilitated in the diagnosis of septic hips [15]. Joint insensitive clinical algorithms do exist for the diagnosis of septic arthritis [16, 17]. Sen et al. (2015) [7] recommend that patients with an acute joint in the outpatient setting receive routine lab work involving serum WBC, ESR, and CRP. Mathews and Coakley (2008) stated that though serum WBC, ESR, CRP, and temperature were all useful in the evaluation of an acute joint, they were not diagnostic [17]. Synovial white cell count is cited as being the only predictive laboratory value, with values $\geq 17,500$ having sensitivity and specificity of $83 \%$ and $67 \%$, respectively, and is considered not better than the "gold standard," which is clinical suspicion by an expert physician with experience in musculoskeletal disease $[16,18]$. Therefore, a more specific set of diagnostic criteria for septic shoulders and increased clinical suspicion would facilitate the clinician in making a prompt diagnosis and thus improve clinical outcomes $[2,3,9,10]$. Additionally, it is important to note that the age of the patients within this study is markedly advanced in comparison to previous literature, and therefore clinicians should have appropriate suspicion for septic arthritis of the shoulder, even in the older child.

\section{Summary}

Septic arthritis of the shoulder is a rare condition. Although generally associated with infants, septic arthritis of the shoulder must be considered when evaluating any patient with shoulder pain and systemic signs of inflammation. We report four cases successfully treated with appropriate antibiotic use and surgical drainage. Septic arthritis of the shoulder may put patients at risk for long-term changes of the articular surface of the joint, and prompt diagnosis and management must be undertaken to reduce the severity of these complications. Development of diagnostic criteria specific to the shoulder would be facilitated in this endeavor and should be addressed in future literature.

\section{Abbreviations}

ESR: Erythrocyte sedimentation rate

WBC: White blood cell

CRP: C-reactive protein.

\section{Disclosure}

Level of evidence is III, retrospective chart analysis.

\section{Competing Interests}

The authors declare that there are no competing interests regarding the publication of this paper.

\section{References}

[1] T. Lejman, M. Strong, P. Michno, and M. Hayman, "Septic arthritis of the shoulder during the first 18 months of life," Journal of Pediatric Orthopaedics, vol. 15, no. 2, pp. 172-175, 1995.

[2] M. H. Perlman, M. J. Patzakis, P. J. Kumar, and P. Holtom, "The incidence of joint involvement with adjacent osteomyelitis in pediatric patients," Journal of Pediatric Orthopaedics, vol. 20, no. 1, pp. 40-43, 2000.

[3] D. Schmidt, S. Mubarak, and R. Gelberman, "Septic shoulders in children," Journal of Pediatric Orthopaedics, vol. 1, no. 1, pp. 67-72, 1981.

[4] E. K. Schallert, J. Herman Kan, J. Monsalve, W. Zhang, G. S. Bisset, and S. Rosenfeld, "Metaphyseal osteomyelitis in children: how often does MRI-documented joint effusion or epiphyseal extension of edema indicate coexisting septic arthritis?" Pediatric Radiology, vol. 45, no. 8, pp. 1174-1181, 2015.

[5] J. M. Melville and T. J. M. Moss, "The immune consequences of preterm birth," Frontiers in Neuroscience, vol. 7, article 79, 2013.

[6] J. A. Ogden, "Pediatric osteomyelitis and septic arthritis: the pathology of neonatal disease," The Yale Journal of Biology and Medicine, vol. 52, no. 5, pp. 423-448, 1979.

[7] E. S. Sen, S. L. N. Clarke, and A. V. Ramanan, "The child with joint pain in primary care," Best Practice \& Research: Clinical Rheumatology, vol. 28, no. 6, pp. 888-906, 2014.

[8] M. Pääkkönen, M. J. T. Kallio, P. E. Kallio, and H. Peltola, “Sensitivity of erythrocyte sedimentation rate and C-reactive protein in childhood bone and joint infections," Clinical Orthopaedics and Related Research, vol. 468, no. 3, pp. 861-866, 2010.

[9] M. V. Belthur, D. L. Palazzi, J. A. Miller, W. A. Phillips, and J. Weinberg, "A clinical analysis of shoulder and hip joint infections in children," Journal of Pediatric Orthopaedics, vol. 29, no. 7, pp. 828-833, 2009.

[10] H.-C. Kao, Y.-C. Huang, C.-H. Chiu et al., "Acute hematogenous osteomyelitis and septic arthritis in children," Journal of Microbiology, Immunology and Infection, vol. 36, no. 4, pp. 260-265, 2003.

[11] K. Gutierrez, "Bone and joint infections in children," Pediatric Clinics of North America, vol. 52, no. 3, pp. 779-794, 2005.

[12] C. O. Montgomery, E. Siegel, R. D. Blasier, and L. J. Suva, "Concurrent septic arthritis and osteomyelitis in children," Journal of Pediatric Orthopaedics, vol. 33, no. 4, pp. 464-467, 2013.

[13] R. Lisbona and L. Rosenthall, "Observations on the sequential use of ${ }^{99 m} \mathrm{Tc}$ phosphate complex and ${ }^{67} \mathrm{Ga}$ imaging in osteomyelitis, cellulitis, and septic arthritis," Radiology, vol. 123, no. 1, pp. 123-129, 1977.

[14] S.-N. Kang, T. Sanghera, J. Mangwani, J. M. H. Paterson, and M. Ramachandran, "The management of septic arthritis in children: systematic review of the English language literature," The Journal of Bone \& Joint Surgery -British Volume, vol. 91, no. 9, pp. 1127-1133, 2009.

[15] M. S. Kocher, D. Zurakowski, and J. R. Kasser, "Differentiating between septic arthritis and transient synovitis of the hip in children: an evidence-based clinical prediction algorithm," The Journal of Bone \& Joint Surgery-American Volume, vol. 81, no. 12, pp. 1662-1670, 1999.

[16] S. F. Li, C. Cassidy, C. Chang, S. Gharib, and J. Torres, "Diagnostic utility of laboratory tests in septic arthritis," Emergency Medicine Journal, vol. 24, no. 2, pp. 75-77, 2007.

[17] C. J. Mathews and G. Coakley, "Septic arthritis: current diagnostic and therapeutic algorithm," Current Opinion in Rheumatology, vol. 20, no. 4, pp. 457-462, 2008.

[18] G. Coakley, C. J. Mathews, M. Field et al., "BSR \& BHPR, BOA, RCGP and BSAC guidelines for management of the hot swollen joint in adults," Rheumatology, vol. 45, no. 8, pp. 1039-1041, 2006. 


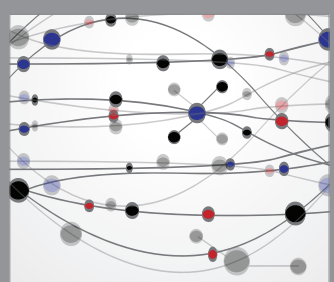

The Scientific World Journal
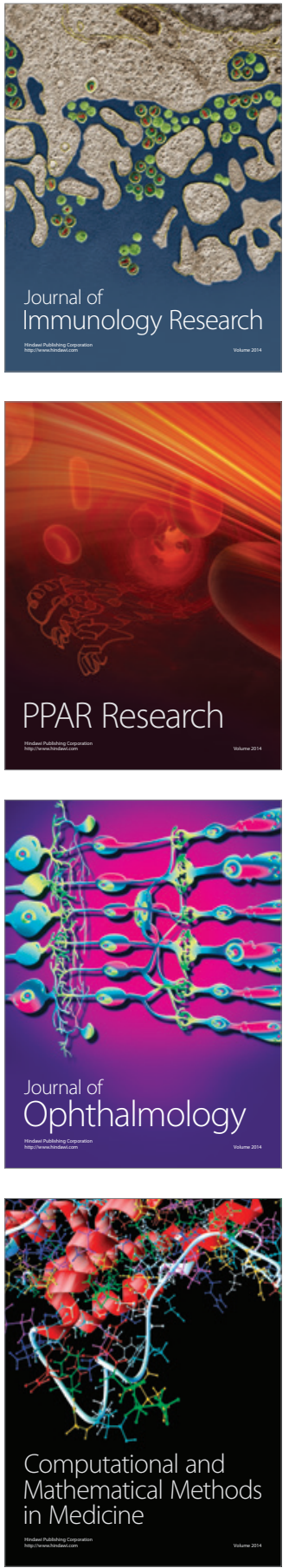

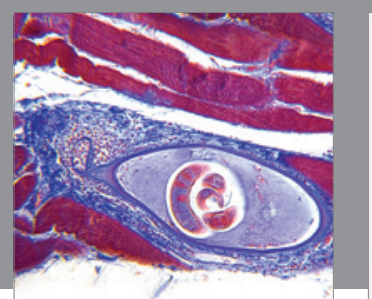

Gastroenterology Research and Practice

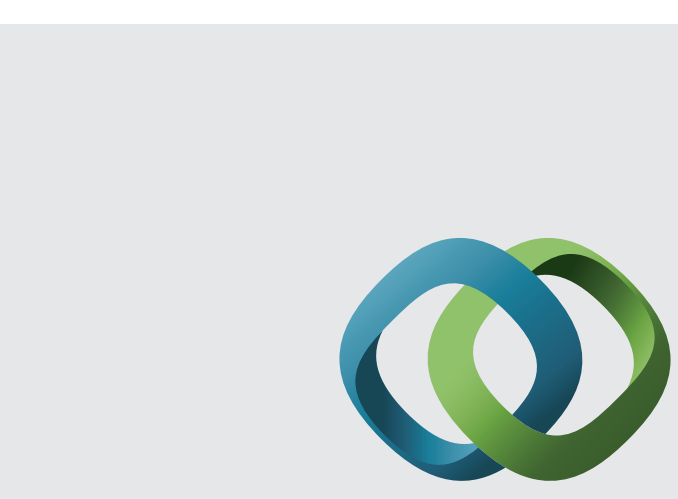

\section{Hindawi}

Submit your manuscripts at

http://www.hindawi.com
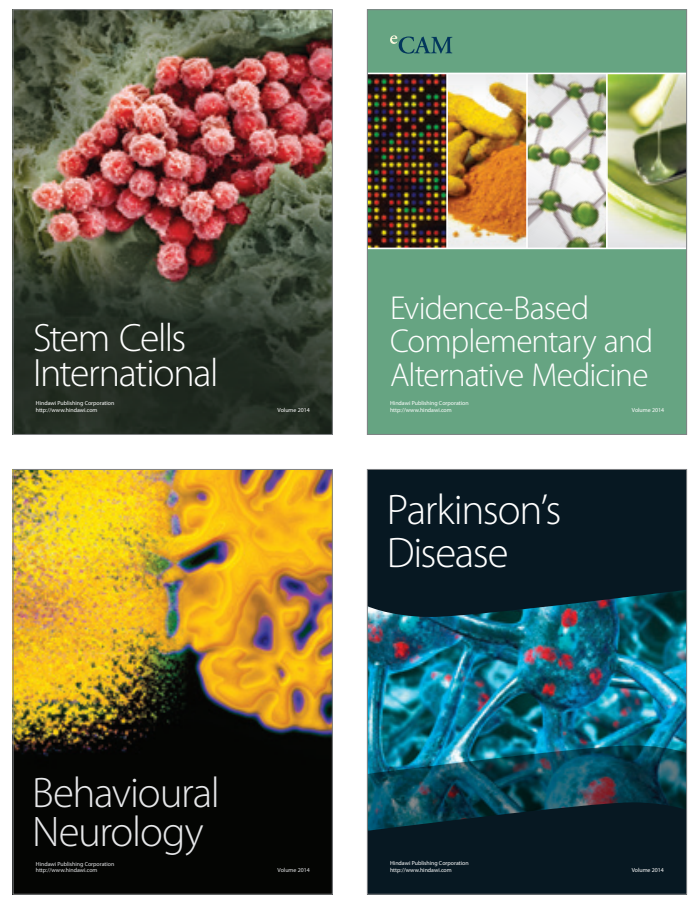
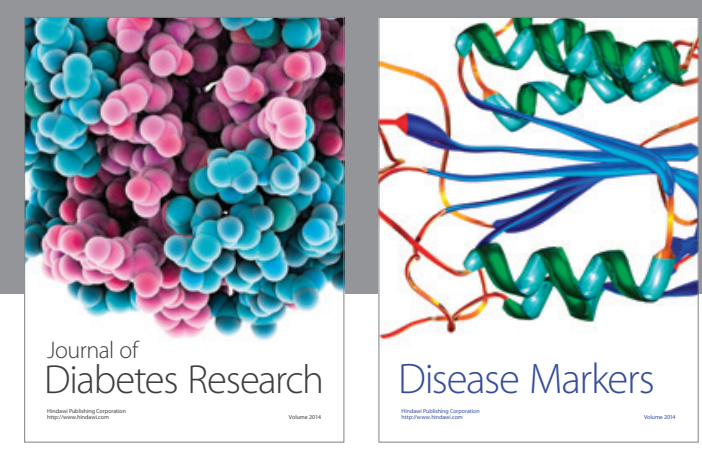

Disease Markers
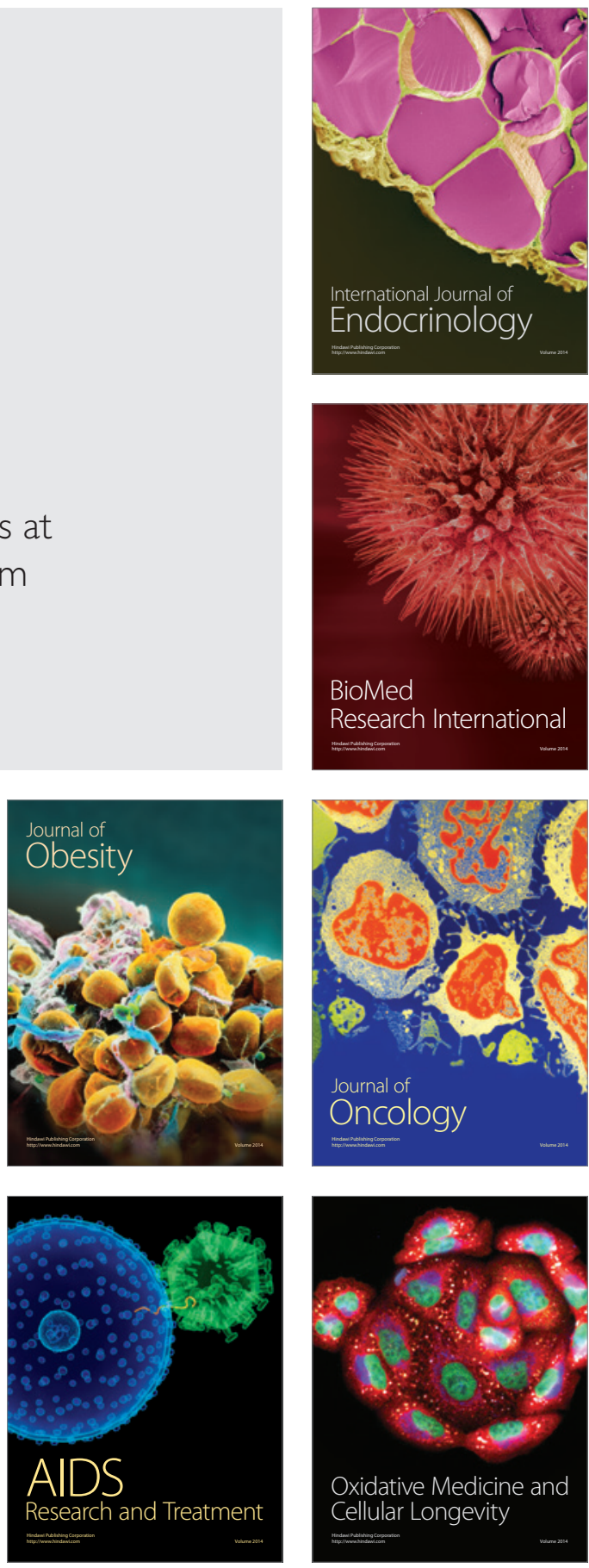